\title{
A rare case of Goldenhar syndrome with radial aplasia*
}

\author{
S. Biswas ${ }^{1}$, A. Adhikari², R. Meyur ${ }^{3}$, P. Kundu ${ }^{4}$ \\ ${ }^{1}$ Department of Anatomy, N.R.S. Medical College, Kolkata, India \\ 2Depatment of Pharmacology, R.G.Kar Medical College, Kolkata, India \\ ${ }^{3}$ Department of Anatomy, R.G.Kar Medical College, Kolkata, India \\ ${ }^{4}$ Department of Anatomy, Bankura Sammilani Medical College, India
}

[Received 15 April 2013; Accepted 10 June 2013]

\begin{abstract}
Goldenhar syndrome (GS) is a well-recognised condition characterised by variable degree of uni- or bilateral involvement of craniofacial structures involving first and second branchial arches manifesting ocular and auricular anomalies and also vertebral defect. This syndrome presents at birth and its exact aetiology is still unknown. The affections of the neural crest cells may have some role in the multiple malformations of the GS. We present a teenaged female case who attended the Orthopaedic Outpatient Department to receive handicapped certificate in Bankura Sammilani Medical College and Hospital. She had multiple skeletal abnormalities, which included hemifacial microsomia, vertebral malformations in the form of scoliosis, distorted pelvis, but the most striking feature was the absence of thumb and aplasia of radius of left side. She did not suffer from any ocular or auricular abnormalities, neither from any cardiological, urogenital or gastroenteric ones. Radial defects associated with GS might represent a subset within this spectrum. Our case probably belongs to this subset, therefore is a very rare one. (Folia Morphol 2013; 72, 4: 362-365)
\end{abstract}

Key words: radial aplasia, Goldenhar syndrome, vertebral defects

\section{INTRODUCTION}

Goldenhar syndrome (GS) is a well-recognised condition characterised by variable degree of unilateral or bilateral involvement of craniofacial structures, involving first and second branchial arches, ocular anomalies and vertebral defect [5]. GS, also known as oculoauriculovertebral dysplasia or hemifacial microsomia, has a wide range of clinical manifestations, including craniofacial, vertebral, cardiac, renal, and central nervous system anomalies [1]. Typical presentation of GS includes epibulbar dermoids, microtia, mandibular hypoplasia, and vertebral anomalies $[8,10,11]$.

GS with radial defects characterises a subset within the syndrome, mainly involving the first branchial arch and limb primordium unilaterally or bilaterally [12].

\section{MATERIALS AND METHODS}

We report an 18-year-old female case who attended the Orthopaedic Outpatient Department in order to receive handicapped certificate in Bankura Sammilani Medical College and Hospital with multiple skeletal abnormalities. The girl was diagnosed to be a case of GS with radial aplasia. The diagnosis was established according to the minimal diagnostic criteria proposed by Stromland et al. [9], considering the combination of hemifacial microsomia, vertebral anomaly and ear or eye malformation.

Address for correspondence: Dr S. Biswas, BJ 145, Sector 2, Salt Lake, Kolkata 700091, India, e-mail: drsharmisthabiswas@rediffmail.com

*The work was carried out in Bankura Sammilani Medical College, Bankura, West Bengal, India. 


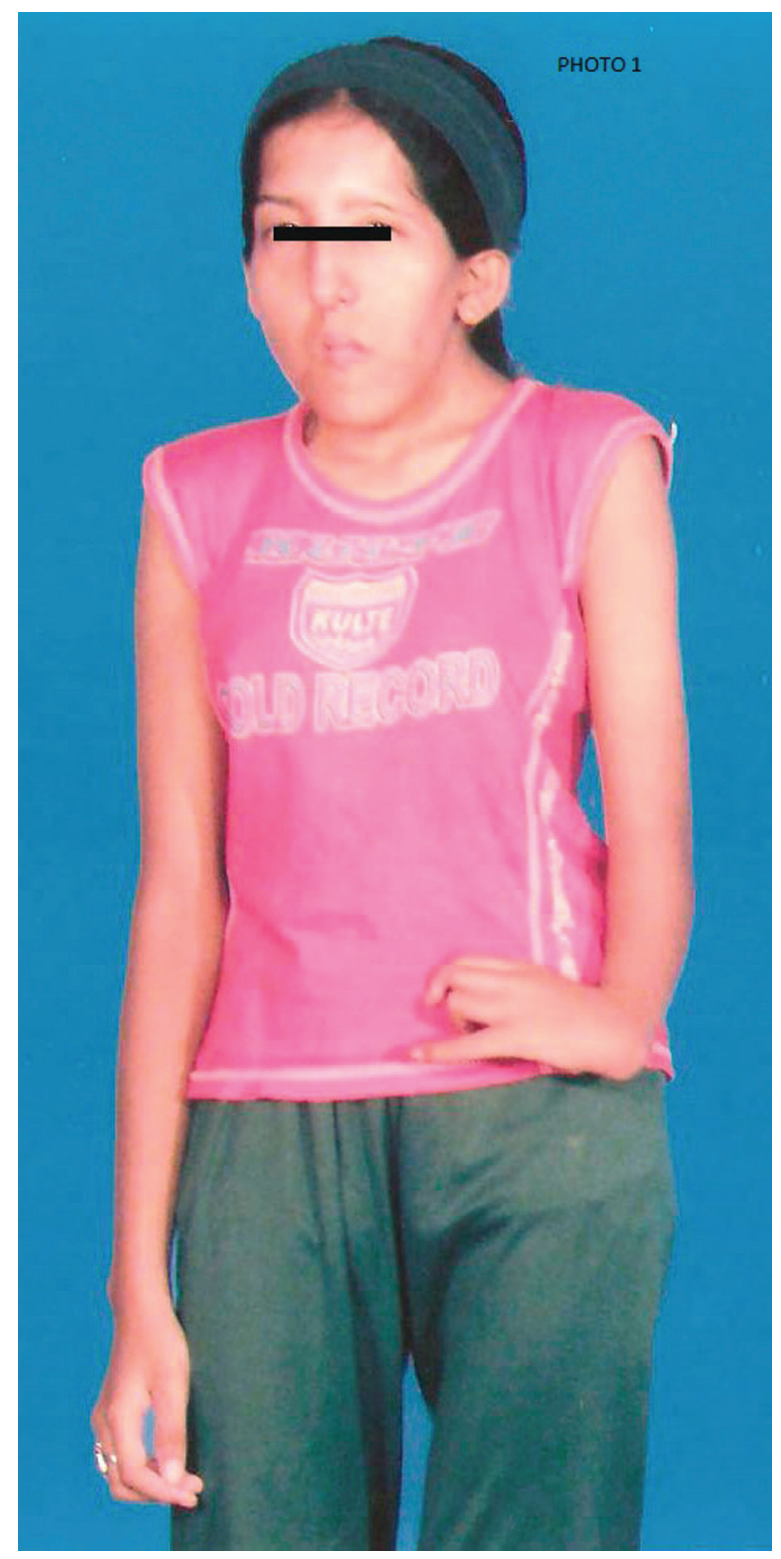

Figure 1. Photograph of the patient showing short left forearm and the absence of thumb on the left side.

Her prenatal, perinatal, and family histories were taken.

The X-ray examinations of head and neck region, upper limbs, lower limbs and hip were performed. The audiometry was carried out. Hematological and cytogenetic studies were done.

\section{RESULTS}

Our case was an 18-year-old female with multiple skeletal abnormalities, which included hemifacial microsomia, facial asymmetry, mandibular hypoplasia, low-set ears, vertebral malformations in the form of scoliosis, distorted pelvis, but the most striking

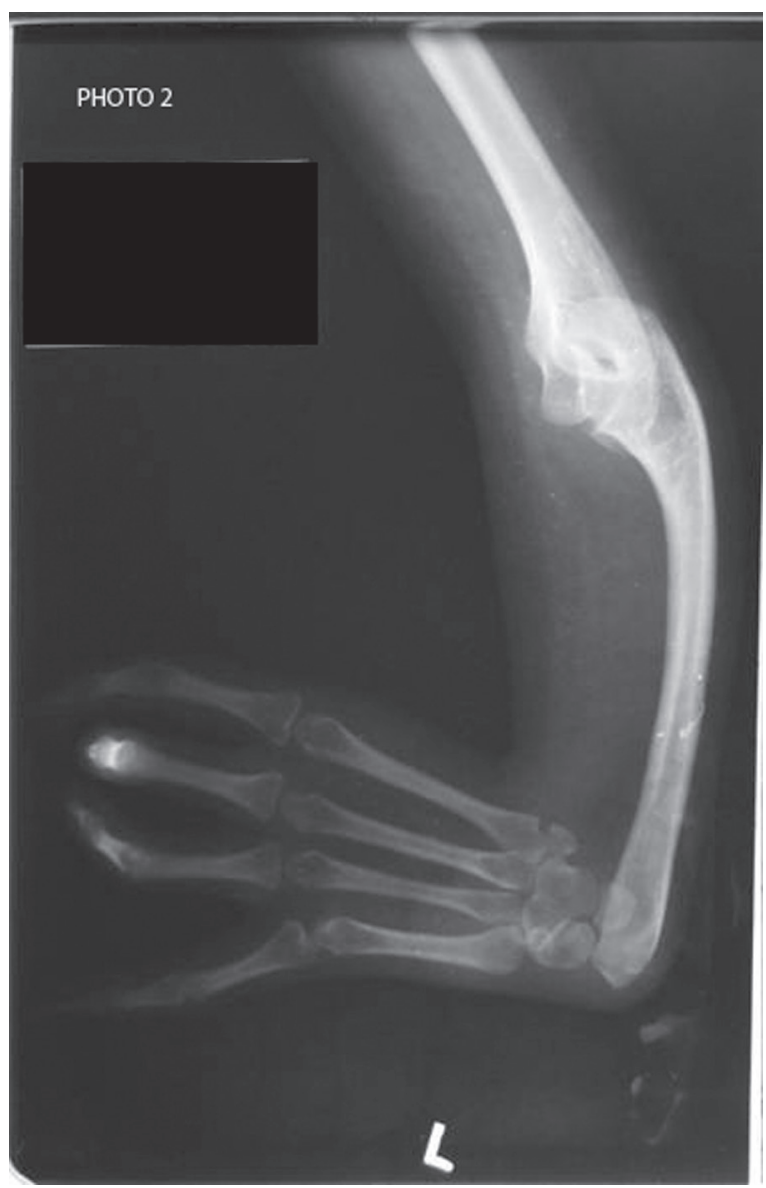

Figure 2. Radiograph of the left upper limb showing the aplasia of radius, the absence of first metacarpal and phalanges of the thumb, and radially placed carpal bones. Ulna is short, hypoplastic and abnormally curved. There are clinodactylies of the medial digits of the left hand.

feature was a short left forearm and the absence of thumb on the left side (Fig. 1). She did not suffer from ocular abnormalities, cardiological, urogenital or gastroenteric abnormalities, nor any hearing defects.

She was the second child of her parents; her elder brother had no abnormalities. The parents were healthy and their marriage was non-consanguineous. The age of the mother was 28 at her birth; the father was 37 years old. She was born by normal vaginal delivery at 34 weeks of gestation. There was no history of exposure to known teratogenic agents, drugs, or maternal diseases. There was no family history for related features.

The X-ray examination of the left upper limb revealed that there was aplasia of radius, absence of first metacarpal and phalanges of the thumb as well as radially placed carpal bones. Ulna was short, hypoplastic and abnormally curved. There were clinodactylies of the medial digits of the left hand (Fig. 2). The X-ray of head and neck region revealed that mandible was 


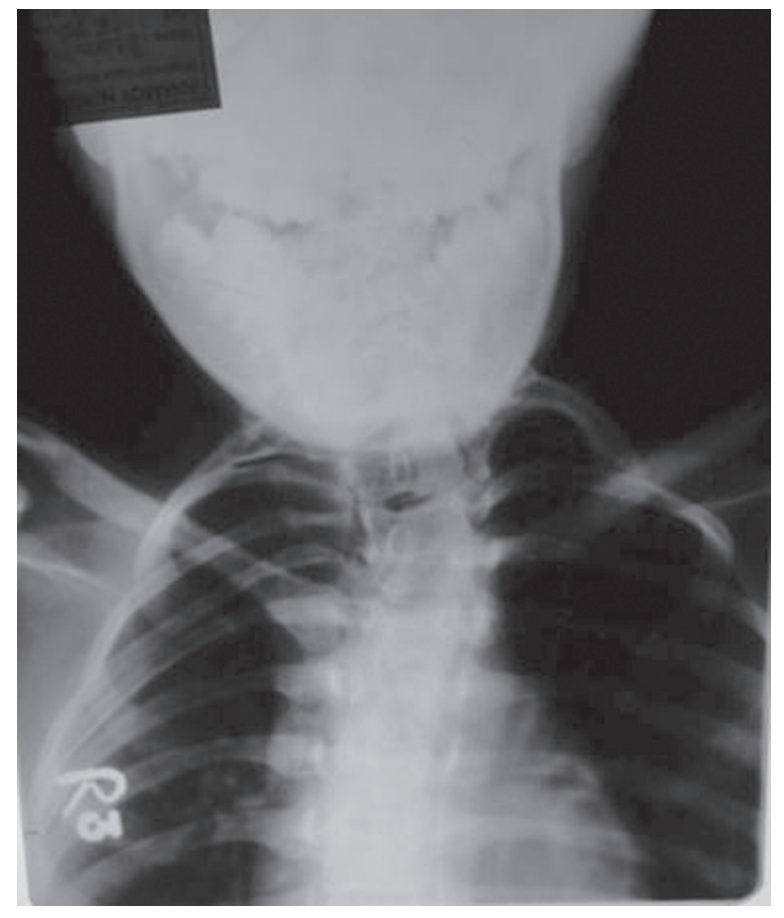

Figure 3. Radiograph showing distorted mandible and misalignments of teeth, and also scoliosis.

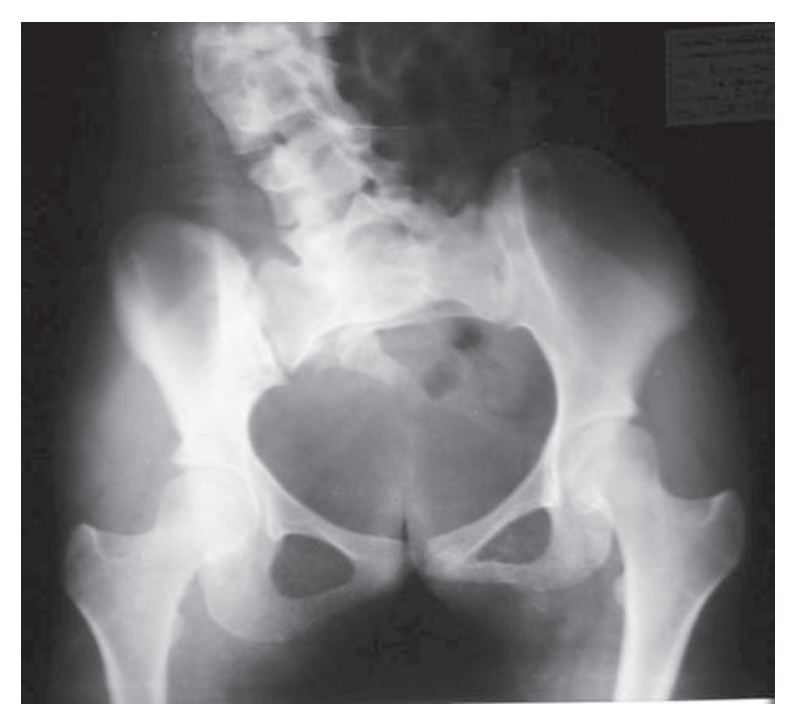

Figure 4. Radiograph revealing scoliosis and distortion of pelvis.

distorted and there were misalignments of teeth; also scoliosis was observed (Fig. 3). Scoliosis and distortion of pelvis were revealed on the $\mathrm{X}$-ray of the hip region (Fig. 4).

No abnormality was detected in the audiometry. Hearing was found to be normal. Hematological and cytogenetic studies were normal.

\section{DISCUSSION}

Interferences in normal development of first and second branchial arches result in variable maxillary, mandibular, and ear abnormalities. These anomalies can be isolated or they can be a part of some known and unknown conditions. Among them is the GS.

Malformations of the external ear or microtia with other anomalies, such as mandible hypoplasia, epibulbar dermoids, and spinal vertebral defects are the features of the syndrome. Cardiac, pulmonary, renal, skeletal, and central nervous system involvements have been observed in patients presented with this condition. Radial defects, although rare, have been reported.

It was postulated by Vendramini et al. [12] that radial defects associated with GS might represent a subset within this spectrum.

There was a neonatal case reported by Digilio et al. [6] with features of facial dysmorphism, ocular coloboma, congenital heart defect, urogenital malformations, and unilateral radial aplasia. The case showed deletion 22 q 11 (del22ql1). In our case, though facial dysmorphism and unilateral radial aplasis were present, there were no cardiac or urogenital malformations. Also, no chromosomal anomalies were found.

Cazabon et al. [3] reported a female case with ocular coloboma and right-sided radial aplasia, absent thumb and only 4 metacarpals. No other congenital abnormalities were detected. Karyotyping using G-banded analysis showed an apparently normal female pattern. No visible cytogenetic abnormality was observed. Our case did not have any ocular defects.

Cetinkaya et al. [4] reported a female infant with micrognatia, low-set ears, facial dysmorphism and unilateral radius aplasia born to a mother who used Lamotrigine and oxcarbazepine during pregnancy for seizures. However, in our case, no drug history of mother or father was available.

Avon and Shively [2] commented that GS - hemifacial microsomia, has been studied extensively with regard to its typical asymmetric craniofacial manifestations, but little attention has been paid to its orthopaedic findings. They reviewed 23 patients with this syndrome with associated orthopaedic abnormalities, which included mainly spinal anomalies, clubfoot, congenital dislocation of the hip, and radial limb defects.

According to Martelli et al. [7], the molecular basis of GS was still unclear; no specific diagnostic tests 
were available. Patients with GS can have multiple congenital anomalies and need particular attention to internal abnormalities. Paediatric specialists should consult with ear-nose-throat, orthopaedics, neurosurgery, and ophthalmology clinics to decide on the most appropriate treatment plan, which varies with age and systemic associations. The possibility of spinal anomalies at all levels of the spine should be considered by those treating cases of GS, since these anomalies cannot be predicted from the severity of facial malformation [1].

\section{CONCLUSIONS}

Radial defects associated with GS might represent a subset within this spectrum. Our case probably belongs to this subset, therefore is a very rare one. The possibilities of orthopaedic involvements must be kept in mind of the physician treating GS.

\section{REFERENCES}

1. Anderson PJ, David DJ (2005) Spinal anomalies in Goldenhar syndrome. Cleft Palate Craniofac J, 42: 477-480.

2. Avon SW, Shively JL (1988) Orthopaedic manifestations of Goldenhar syndrome. J Pediatr Orthop, 6: 683-686.

3. Cazabon S, Bradbury JA (2003) Ocular coloboma and radial aplasia:syndrome or association. Eye, 17: 532-534.

4. Cetinkaya M, Ozkan H, Köksal N (2008) Unilateral radius aplasia due to lamotrigine and oxcarbazepine use in pregnancy. J Matern Fetal Neonatal Med, 21: 927-930.
5. Cohen MM Jr, Rollnick BR, Kaye Cl (1989) Oculoauriculovertebral spectrum: an updated critique. Cleft Palate J, 26: $276-286$.

6. Digilio M C, Giannotti A, MarinoB, Guadagni AM, Orzalesi M, Dallapiccola B (1997) Radial aplasia and chromosome 22ql 1 deletion. J Med Genet, 34: 942-944.

7. Martelli H Jr, Miranda RT, Fernandes CM, Bonan PR, Paranaíba LM, Graner E, Coletta RD (2010) Goldenhar syndrome: clinical features with orofacial emphasis. J Appl Oral Sci, 18: 646-649.

8. Rollnick BR, Kaye Cl, Nagatoshi K, Hauck W, Martin AO (1987) Oculoauriculovertebral dysplasia and variants: phenotypic characteristics of 294 patients. Am J Med Genet, 26: 361-375.

9. Stromland K, Miller M, Sjogreen L, Johansson M, Joelsson BM, Billstedt E (2007) Oculo-auriculo-vertebral spectrum: associated factors. Am J Med Genet A, 143A: 1317-1325.

10. Tasse $C$, Bohringer $S$, Fischer $S$, Ludecke $H J$, Albrecht $B$, Horn D, Janecke A, Kling R, Konig R, Lorenz B, Majewski F, Maeyens E, Meinecke P, Mitulla B, Mohr C, Preischl M, Umstadt H, Kohlhase J, Gillessen-Kaesbach G, Wieczorek D (2005) Oculo-auriculo-vertebral spectrum (OAVS): clinical evaluation and severity scoring of 53 patients and proposal for a new classification. Eur J Med Genet, 48: 397-411.

11. Touliatou V, Fryssira H, Mavrou A, Kanavakis E, Kitsiou-Tzeli S (2006) Clinical manifestations in 17 Greek patients with Goldenhar syndrome. Genet Couns, 17: 359-370.

12. Vendramini S, Richieri-Costa A, Guion-Almeida ML (2007) Oculoauriculovertebral spectrum with radial defects: a new syndrome or an extension of the oculoauriculovertebral spectrum? Report of fourteen Brazilian cases and review of the literature. Eur J Human Gen, 15: 411-421. 